\title{
Persistence of Seismic Rockmass Response to Mining
}

\author{
A.J. Mendecki ISS International Limited, South Africa
}

Mining is not a spontaneous process. It induces stresses in the rockmass at particular place, at particular time and at particular rate, influencing the development of spatial and temporal correlations and interfering with the act of selforganisation. Some particular mining scenarios may therefore generate more favorable short term seismic response than others. It has been shown in one example of tabular sequential grid mining that periods of non-contiguous blasting of mining panels were associated with lower hazard than when blasting was done contiguously, irrespective of the rate of mining. Non-contiguous blasting at lower mining rate generated even more favorable conditions. These results confirmed previous observations of seismic rockmass response to production blasting associated with long-wall mining (Mendecki, 2001). The data sets also show the presence of long-term correlations, indicating the tendency for the local trend of lower seismic hazard to persist. It may be that mining scenarios that induce spatial heterogeneity prevent smoothing, de-correlate the system and are less likely to develop larger dynamic instabilities.

\section{INTRODUCTION}

One of the interesting concepts in the interpretation of seismic activity is the notion of self-organisation into subcritical state, state at which the correlation length becomes comparable with the system size and at which the system can develop and maintain reproducible relationships among its distant parts. It is assumed that the growth of long-range correlations within the rockmass allow for progressively larger events to be generated. Intermittently, correlations may reach or even exceed the size of the observed area, creating conditions conducive for large instabilities. An important agent in the development of spatial and temporal correlations is seismic activity itself. By breaking numerous asperities, seismic events smooth the system, allowing transfer of stresses over larger distances and thus paving the way for even larger events. Disorder, on the other hand, may play stabilizing role and, to degree, can be engineered into the system by scattered layout and/or by scattered sequence of mining or blasting. Disordered directions of local stresses and low loading rate, induced by the scattered mining environment, may also play role, since it promotes healing and thus stress roughening.

The objective of this paper is to demonstrate the differences in the short term seismic rockmass response to orderly, or contiguous, and disorderly, or non-contiguous, production blasting in deep level tabular sequential grid mining scenario. In addition the data set tests positive for the existence of longterm correlations, indicating the tendency for muted seismic response associated with non-contiguous and/or low rate mining to persist. For completeness, some consideration is given to the description of seismic potency, magnitude scales, the potency-frequency distribution and to the rescaled analysis.

\section{SEISMIC POTENCY AND MAGNITUDE}

Charles Richter observed that the logarithm of peak ground motion decayed with distance along parallel curves for different earthquakes (Richter, 1935). Thus, for an event with amplitude of displacement $\AA$, the difference $\log \AA-\log \AA_{0^{\prime}}$ would measure its relative size to a reference event with amplitude $\AA_{0}$. Richter selected the reference earthquake with $\AA_{0}=10^{-3}$ meters recorded at the epicentral distance of $100 \mathrm{~km}$ and called it the local magnitude $m_{L}=0.0$. All recordings were made with the narrow band Wood-Anderson seismometer with approximately 0.8 second period, so the maximum amplitude was always at the same frequency, and it applied to shallow earthquakes in California recorded at distances of 30 to $600 \mathrm{~km}$. The local magnitude then is relative measure of the strength of seismic event and unit increase in magnitude corresponds to 10 fold increase in the amplitude of ground displacement. To be applied outside California the local magnitude scale requires calibration.

The magnitude scale was later extended by the introduction of surface-wave magnitude, $m_{S}=\log \AA+1.656 \log \Delta+1.818$, where $\AA$ is the maximum horizontal component of the surface waves, with period of about 20s, in micrometers and $\Delta$ is the epicentral distance; and body wave magnitude, $m_{B}=$ $\log (\AA / T)+q(\Delta, h)$, where $T$ is the period associated with the maximum body wave amplitude and $q(\Delta, h)$ is the calibrating function to correct for path, including depth $h$, and site effects (Gutenberg, 1945a; Gutenberg, 1945b). There is also duration magnitude, based on the assumption that the coda amplitude is function of time $t_{F-0}$ measured between the origin time of an event and the tail end of its coda waves, $m_{t}=2.8 \log t_{F-0}+$ constant (Aki, 1969; Real and Teng, 1973; Aki, 1987).

The underlying intention of different magnitude definitions was to offer an equivalent measure to earthquakes radiating the same amount of energy. Today, the direct estimate of magnitude is performed routinely by national or regional seismological networks equipped with the low frequency sensors able to quantify larger earthquakes.

In most areas in the world the range of magnitudes of seismic events recorded in mines is not well covered by the regional or national seismological networks and it is difficult to calibrate their records against local magnitudes. Since the modern seismic systems deployed in mines derive source parameters from recorded waveforms, one can try to scale them with magnitude.

Gutenberg and Richter (1956) derived an empirical relationship between the radiated seismic energy $E$ and the surface-wave magnitude of larger earthquakes 


$$
\log E=1.5 \mathrm{~m}+4.8
$$

or

$$
m=\frac{2}{3} \log E-3.2
$$

According to equation (1) unit increase in magnitude corresponds to an approximately 30 times increase in radiated energy. Although the relation (1) is empirical, the implied scaling $m \sim \frac{2}{3} \log E$, can be explained for most moderate to large earthquakes in terms of simple dislocation model with constant stress drop, while very small earthquakes are likely to satisfy $m \sim \log E$ (Haskell, 1964; Kanamori and Anderson, 1975). Since the determination of radiated seismic energy depends on the frequency bandwid th and the sensitivity of the monitoring system and because the frequency distribution of seismic waves changes with event size (Aki, 1967), the energy based magnitude scale (2) struggles to represent small and large events adequately.

A more reliable and easy to determine parameter pertaining to the source is seismic potency $P=\Delta \varepsilon V$ in $\left[\mathrm{m}^{3}\right]$, where $\Delta \varepsilon$ is the strain change at the source and $V$ the source volume or, for a planar source, $P=\bar{u} \Lambda$ in $\left[\mathrm{m} \cdot \mathrm{m}^{2}\right]$, where $u$ is an average displacement and $A$ the source area (King, 1978; Ben-Menahem and Singh, 1981). Potency is most frequently estimated from the amplitude of the low frequency displacement spectra $\Omega_{0}$ of the recorded waveforms (Keilis-Borok et al., 1960)

$$
P_{(P, S)}=4 \pi v_{(P, S)} R \frac{\Omega_{0,(P, S)}}{\Lambda_{(P, S)}}
$$

where $v_{(P, S)}$ is $\mathrm{P}$ or $\mathrm{S}$-wave velocity, $R$ is the distance from the source and $\Lambda_{(P S)}$ is the root-mean-square value for the radiation pattern of far-field amplitudes averaged over the focal sphere: $\Lambda_{P}=0.516$ for P-waves and $\Lambda_{S}=0.632$ for $\mathrm{S}$-waves. Seismic potency is the primary parameter that can be derive from waveforms and it provides proper physical measure for the overall size of seismic event. Seismic moment is the product of rigidity $\mu$ and potency, $M=\mu P$ and it is an ambiguous measure for sources occurring at rock interfaces with different rigidities or for sources in thin layer of low rigidity, e.g. fault gouge, sandwiched in stiff rock (Heaton and Heaton, 1989). Since the linear elastic waves generated by shear dislocation have no information on material properties at the source, the potency is an objective observable scaling parameter for the size of an event (Ben-Zion, 2001).

The energy available for seismic radiation during shear dislocation $u$ over the area $A$ driven by the average stress $\sigma$ can be approximated by $E=\xi \sigma P=\xi \sigma M / \mu$, where $\xi$ is the seismic efficiency. The product $\xi \sigma$ is called apparent stress, $\sigma_{A}=E / P$, (Wyss and Brune, 1968), which is considered model independent measure of stress release at the source. Seismic sources similar in terms of their potency may differ by up to two orders of magnitude in radiated energies, reflecting stress differences at the place and at the time of their occurrence (Mendecki, 1993). Comparing $E=\sigma_{A} P=\sigma_{A} M / \mu$ with equation (2) gives

$$
m=\frac{2}{3} \log P+\frac{2}{3} \log \left(\sigma_{A}\right)-3.2
$$

which, for the apparent stress of 15 bars $=15 \cdot 10^{5} \mathrm{~Pa}$ and $\mu=30 \mathrm{GPa}$, gives

$$
m=\frac{2}{3} \log P+0.92=\frac{2}{3} \log M-6.06
$$

Equation (5) is known as the moment-magnitude relation (Kanamori and Anderson, 1975; Kanamori, 1977; Hanks and Kanamori, 1979) and it is calibrated so that it is close to the surface wave magnitude $m_{s}$. Similar relations between seismic moment and local magnitude were obtained earlier by Wyss and Brune (1968), Aki (1969) and Thatcher and Hanks (1973). Equations (4) and (5) assume constant apparent stress, in this particular case $\log E=\log P+6.176$, which implies slope of 1.0 on the $\log E$ vs $\log P$ plot, which is not always supported by data. In more general case

$$
\log E=d \log P+c
$$

where $d$ is the slope and the $c$ intercept that measures the log $E$ released by seismic event with $\log P=0$. In such case apparent stress scales with potency as $\log \sigma_{\mathrm{A}}=(d-1) \log P+c$, and for $d=1$ apparent stress is independent of potency, $\sigma_{\mathrm{A}}=10^{c}$. For $d>1$ apparent stress would increase with an increase in seismic potency. By combining equation (1) with (6) one obtains more general magnitude-potency relation

$$
m=\frac{2}{3} d \log P+\frac{2}{3} c-3.2
$$

or

$$
P=10^{(1.5 m-c+4.8) / d}
$$

The slope $d$ of the $\log E v s \log P$ plot of events recorded in hard rock mines is frequently reported to be higher than 1.0. Spottiswoode and McGarr (1975) calculated seismic moments, radiated energies and magnitudes of 24 events associated with deep level mining on the East Rand in SA from analog waveforms recorded at surface site above the mine. They confirmed the relation (1) for events in the magnitude range $0 \leq m \leq 3$ and reported $\log M=1.2 m+10.7$ which, for $\mu=30 \mathrm{GPa}$, gives $m=0.833 \log P-0.186$ and, together with equation (1), gives $\log E=1.25 \log P+4.52$. Mendecki (1993) and van Aswegen and Butler (1993) analysed high dynamic range digital waveforms of thousands of events in the range, $-0.5 \leq m \leq 3.5$, recorded underground on West Rand, Klerksdorp and Welkom gold mines. They reported $\log E=1.5( \pm 0.1) \log (\mu P)-10.5( \pm 0.5)$ which, for $\mu=30 \mathrm{GPa}$, gives $\log E=1.5 \log P+5.22$ and $m=\log P+0.28$. A similar relation, $m=\log P+0.72$, was obtained by Ben-Zion and Zhu (2002) for small earthquakes, $m<3.5$, recorded by Abercrombie (1996) with $4.5 \mathrm{~Hz}$ sensors located in a deep borehole in California. For data sets with larger events, $m>3.5$, recorded by the TERRAscope/ TriNet network they obtained $m=1.34 \log P+1.22$. The local magnitudes used by Ben-Zion and Zhu (2002) were determined independently by the Southern California ShortPeriod Network.

If the observed energies and potencies scale according to equation (6), one can estimate magnitudes from equation (7). Alternatively one can simply use $\log P$ as measure of magnitude - see Table $1 . \log P$ is simple, reliable, independent of rigidity and portable, therefore one can objectively compare events and seismic hazard between different mines.

\section{POTENCY-FREQUENCY DISTRIBUTION}

Combining the magnitude-frequency relation, $\log N(\geq m)=$ $a-b m$, with equation (7), gives the following frequencypotency distribution

$$
\log N(\geq P)=\log \alpha-\beta \log P
$$

that can be written as

$$
N(\geq P)=\alpha P^{-\beta}
$$

where $N(\geq P)=$ is the number of events with seismic potency not smaller than $P, \alpha=10^{a-2 b(c-4.8) / 3}$ and $\beta=\frac{2}{3} d b$. The parameter $\alpha$ measures the number of events with potency 
TABLE 1 Magnitudes calculated according to different potency-magnitude relations discussed in the text versus the selected logarithms of seismic potency, $\log P$ : Hanks-Kanamori (H-K), Spottiswoode and McGarr (S-McG), Mendecki, van Aswegwen and Butler (M-vA-B) and Ben-Zion and Zhu (BZ-Z)

\begin{tabular}{lcccc}
\hline & \multicolumn{4}{c}{ magnitude relation } \\
logP & H-K & S-McG & M-vA-B & BZ-Z \\
\hline-2 & -0.4 & -1.8 & -1.7 & -1.3 \\
-1 & 0.3 & -1.0 & -0.7 & -0.3 \\
0 & 0.9 & 0.2 & 0.3 & 0.7 \\
1 & 1.6 & 0.6 & 1.3 & 1.7 \\
2 & 2.3 & 1.5 & 2.3 & 2.7 \\
3 & 2.9 & 2.3 & 3.3 & 3.7 \\
4 & 3.6 & 3.1 & 4.3 & 4.7 \\
\hline
\end{tabular}

not smaller than one, $\alpha=N(P \geq 1)$. The single largest expected event would have a potency $P_{\max }$ that corresponds to $\log 1=$ $\log \alpha-\beta \log P_{\max }$, thus

$$
P_{\max }=\alpha^{1 / \beta}=P_{\min }\left[N\left(\geq P_{\min }\right)\right]^{1 / \beta}
$$

where $P_{\min }$ is the minimum potency above which the data set is complete and defines the sensitivity of the monitoring system. The mean recurrence time of events with potency not smaller than $P$ is

$$
\overline{\mathrm{t}}(\geq P)=\frac{\Delta t}{N(\geq P)}=\frac{\Delta t}{\alpha P^{-\beta}}
$$

where $\Delta t$ is the period of observation. The equation (10) represents power law distribution of $P$. Similar power law relations may be derived for the radiated seismic energy $E$ and for the source area $A$. power law is an example of "wild" or "fat-tail" distributions, where large events are much more likely to occur than if the distribution was "mild" or "thin-tail", e.g. exponential. A power law also implies scale invariance or the lack of a characteristic scale and, as consequence, puts no prior limit on the maximum event size.

In general the $\beta$-value is influenced by rockmass and stress heterogeneity, the level of stress and the stiffness of the system under consideration. The rockmass heterogeneity depends on the spatial distribution of sizes, and distances between, strong and/or stressed and weak and/or de-stressed patches of rock where seismic sources may be stopped and nucleate. In general, for the same stiffness, an increase in rock heterogeneity results in higher $\beta$-value, since it is more likely that an initiated rupture be stopped by soft or hard patch before growing into larger event (Mogi, 1962; Izutani, 1984; Mori and Abercombie, 1997)

The explanation that $\beta$-values tend to be lower in high stress areas is based on similar reasoning, namely, that rupture once initiated grows larger in high stress environments. If one assumes that the time of rupture of larger events is controlled by strong and highly stressed patches within the rockmass, seismic hazard should then be calculated on the basis of magnitude-frequency data selected from these areas only, as determined by the contours of energy index, seismic stress or modeled stress, instead of from the entire volume (Wiemer and Wyss, 1997).

Stiffness can be described as the ability of the system to resist deformation with increasing stress. The stiffer the loading system the higher the $\beta$-value (van Aswegen and Mendecki, 1999; Mendecki et al., 1999). This observation does not contradict reports on decreasing $\beta$-value with increasing stress (Scholz, 1968), since there is general loss of stiffness with increasing stress during strain hardening regime. Experimental studies on rock samples in triaxial conditions show decrease in $\beta$-value of acoustic emission events with both the differential stress and with the confining pressure during all stages of stress-strain behavior, including the post peak strain softening regime (e.g. Amitrano, 2003). In the absence of significant tectonic stress, intermediate and large seismic events usually occur after considerable mining has taken place in the area, degrading the stiffness of the system.

From (10) the cumulative distribution function, $F(P)$, and the probability of having an event with potency not greater than $P \geq P_{\min }$ is

$$
\operatorname{Pr}(\leq P)=F(P)=\frac{N(\leq P)}{N\left(\geq P_{\min }\right)}=1-P_{\text {min }}^{\beta} P^{-\beta}
$$

The probability density function of potency frequency distribution $f(P)$ is defined as

$$
f(P)=\frac{d}{d P} F(P)=\beta P_{\min }^{\beta} P^{-\beta-1}
$$

The probability of having a seismic event within the potency range $\left(\mathrm{P}_{1}, \mathrm{P}_{2}\right)$ is

$$
\operatorname{Pr}\left(P_{1}, P_{2}\right)=P_{\text {min }}^{\beta}\left(P_{1}^{-\beta}-P_{2}^{-\beta}\right)
$$

and the potency produced in this range can be calculated from

$$
P\left(P_{1}, P_{2}\right)=N \int_{P_{1}}^{P_{2}} P f(P) d P
$$

where $N=N\left(\geq P_{\text {min }}\right)=\alpha P_{\text {min }}^{-\beta}$. For $\beta \neq 1$ the integral (16) gives

$$
P\left(P_{1}, P_{2}\right)=\frac{\alpha \beta}{1-\beta}\left(P_{2}^{1-\beta}-P_{1}^{1-\beta}\right)
$$

and for $\beta=1$

$$
P\left(P_{1}, P_{2}\right)=\alpha \ln \frac{P_{2}}{P_{1}}
$$

For $\beta<1$, the $\lim _{P \rightarrow 0} P^{1-\beta}<\infty$, and one can consider extrapolating the potency produced below the $P_{\text {min }}$, from $P \rightarrow 0$ to $P_{2}<\infty$

$$
P\left(0, P_{2}\right)=\frac{\alpha \beta}{1-\beta} P_{2}^{1-\beta}
$$

For $\beta>1$, the $\lim _{P \rightarrow \infty} P^{I-\beta}<\infty$, and one can extrapolate seismic release from the finite $P_{1}>0$ to $P_{2} \rightarrow \infty$

$$
P\left(P_{1}, \infty\right)=\frac{-\alpha \beta}{1-\beta} P_{1}^{1-\beta}
$$

It is obvious now that the power law distribution with a given exponent $\beta<1$ that holds for small and intermediate events can not be extended with the same exponent to infinity, because it would require that an infinite amount of potency be produced from the rockmass. If the distribution for the largest events is indeed power law then it must have an exponent $\beta>1$.

For $\beta<1$, the following ratio quantifies the portion of seismic inelastic deformation below $P_{\min }$ that, if already produced by the rockmass, is missing because it could not have been recorded due to the limited sensitivity of the seismic monitoring system or, it has been produced aseismically

$$
\frac{P\left(0, P_{\min }\right)}{P\left(P_{\min }, P_{\max }\right)}=\frac{P_{\min }^{1-\beta}}{P_{\max }^{1-\beta}-P_{\min }^{1-\beta}}
$$


In general, the ratio (21) increases with an increase in the $\beta$-value and with decrease in the range $P_{\max }-P_{\min }$. Theoretically the missing portion can be as low as fraction of percent for large range potency and low $\beta$ or, it may be quite considerable in the opposite case - see Figure 1.

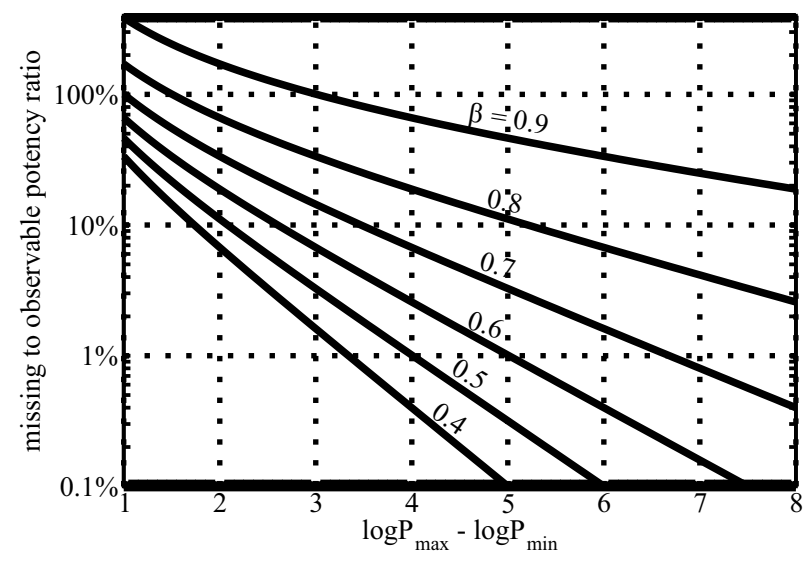

FIG. 1 The portion of potency below the threshold of the monitoring system, $P\left(0, P_{\min }\right)$, as percentage of the total observable potency, $\left(\boldsymbol{P}_{\min }, \boldsymbol{P}_{\max }\right)$, shown here as function of the observable potency range, $\log P_{\max }-\log P_{\min }$ - see equation (21)

\section{EFFECTIVE VOLUME MINED AND POTENCY- FREQUENCY DISTRIBUTION}

Suppose that given volume of rock, $V_{m}\left(t_{0}\right)$, is mined out at the time $t_{0}$. Then if, in the absence of other loading, the altered stress and strain field can readjust to an equilibrium state through seismic deformation only, the sum of potency, $\Sigma P\left(t_{j}\right)$, produced by number of events at times $t_{i} \geq t_{0}$ would be less or, in the long run, equal to $V_{m}$ (McGarr, 1976a),

$$
\sum_{t_{j} \geq t_{0}} P\left(t_{j}\right) \leq V_{m}\left(t_{0}\right)
$$

If the extraction of rock takes place at discrete times, as does the backfill placement and seismic deformation, then one can construct the following balance step function $B(t)$,

$$
B(t)=V_{e f f}(t)-\sum_{t_{k} \leq t} P\left(t_{k}\right) \Theta\left(t-t_{k}\right)
$$

where $V_{\text {eff }}(t)=\Sigma_{t_{i} \leq t} V_{m}\left(t_{i}\right) \Theta\left(t-t_{i}\right)-\Sigma_{t_{j} \leq t} V_{b}\left(t_{j}\right) \Theta\left(t-t_{j}\right), V_{m}\left(t_{i}\right)$ is the volume mined at the time of $t_{i}, V_{b}\left(t_{j}\right)$ is the effective volume of backfill placed at the time $t$, corrected for shrinkage and compaction, $P\left(t_{k}\right)$ is the potency of the event that has occurred at the time $t$ and $\Theta$ is the Heaviside step function, $(\Theta(t)=0$ if $\mathrm{t}<0$ and $\Theta(t)=1$ if $t \geq 0$ ). The trend, the change of rate, the periodicity and the deficit of $B(t)$ may be indicative of the capacity for seismic release. At any given time $t$, the second term of the right hand side of equation (23) can be approximated by the integral (19), which for $P_{1}=0, P_{2}=P_{\operatorname{maxm}}$ and $\beta<1$, gives the finite amount of potency

$$
\sum_{t_{k} \leq t} P\left(t_{k}\right) \Theta\left(t-t_{k}\right)=\frac{\alpha \beta}{1-\beta} P_{\max }^{1-\beta}
$$

Assuming: $B(t)=0, \beta<1$, and solving equations (23) and (24) for $P_{\operatorname{maxm}}$ gives

$$
P_{\max m}\left[V_{e f f}(t)\right]=\left[\frac{1-\beta}{\alpha \beta} \cdot V_{e f f}(\mathrm{t})\right]^{\frac{1}{1-\beta}}
$$

The relation (25) estimates the maximum possible event size $P_{\operatorname{maxm}}$ that, given the parameters of the size distribution and $\alpha$ and $\beta$ the effective volume mined $V_{e f f}$ would close the deficit of deformation. The interpretation of equation (25) is straightforward: $P_{\operatorname{maxm}}$ increases with $V_{\text {eff }}$ and, for a given $V_{\text {eff }}$ the more potency that has been produced to date, meaning higher $\alpha$ and lower $\beta$ in the potency-frequency distribution, the less is to be released to close the deficit, therefore the $P_{\text {maxm }}$ gets lower. Scaling, for a given $V_{\text {eff }}$ follows similar logic: for $\beta$ as low as $1 / 3$, the maximum possible potency scales with the effective volume mined as $P_{\operatorname{maxm}} \sim V_{\text {eff }}^{3 / 2}$, for $\beta=1 / 2$, $P_{\text {maxm }} \sim V_{\text {eff }}^{2}$ and, for $\beta=2 / 3, P_{\text {maxm }} \sim V_{\text {eff }}^{3}$.

Relations (23) and (25) are based on few assumptions that may limit their applications. Firstly, they imply that one knows the total potency produced due to mining in given area. In practice, however, one can at best account only for that portion of seismic potency which has been released since the introduction of the monitoring system. Secondly, they assume that all inelastic deformation is seismic, or that it can be derived from seismic measurements. In reality, seismic system measures only that portion of the inelastic deformation which is associated with seismic radiation and which is within the limits of its sensitivity. Extrapolating the potency-frequency relation down to zero by means of equation (21) does not account for all aseismic deformation. Thirdly, the relation (25) assumes that the only stress acting in the area is the one induced by the particular volume mined, which may not be the case in scattered mining scenarios or in the presence of tectonic stresses.

Figures 2 to 5 and Table 2 document an example of seismic response to different ways of production blasting at section of the Mponeng mine, where high resolution seismic and production data are available for the period 01Sept'03 to $15 \mathrm{Feb}^{\prime} 04$.

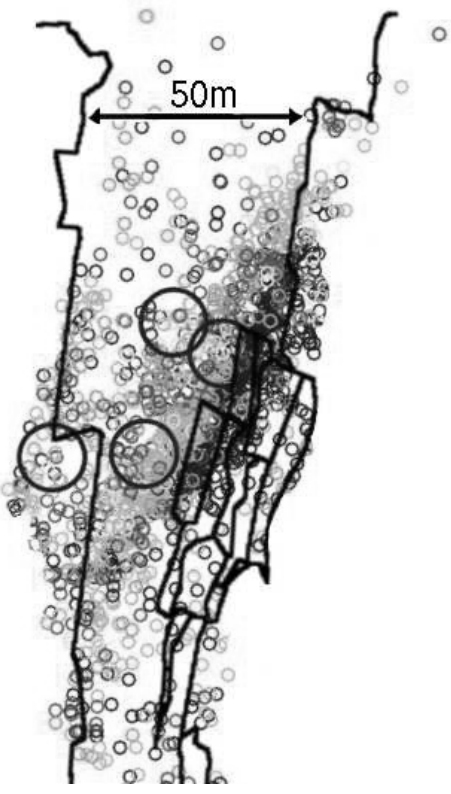

FIG. 2 The plan view of the area at the Mponeng mine showing panels progressing west (to the left). Circles are seismic events recorded between 1 Sept'03 and 15 Feb'04.

Spheres are the four largest events with $\log P=1.2,1.1$, $1.2,1.0$ respectively, that occurred in this area within the period 15 Feb to 05 June'04

The entire time span was divided into 6 periods on the basis of the type of production blasting (contiguous or non-contiguous), stoppages of production and the timing of the largest events (preferably at the end of the period). The first and the sixth periods are associated with production by blasting predominantly non-contiguous panels, the fifth 
period coincides with Christmas with hardly any mining, while during other periods blasting was mainly contiguous - see Figure 3.

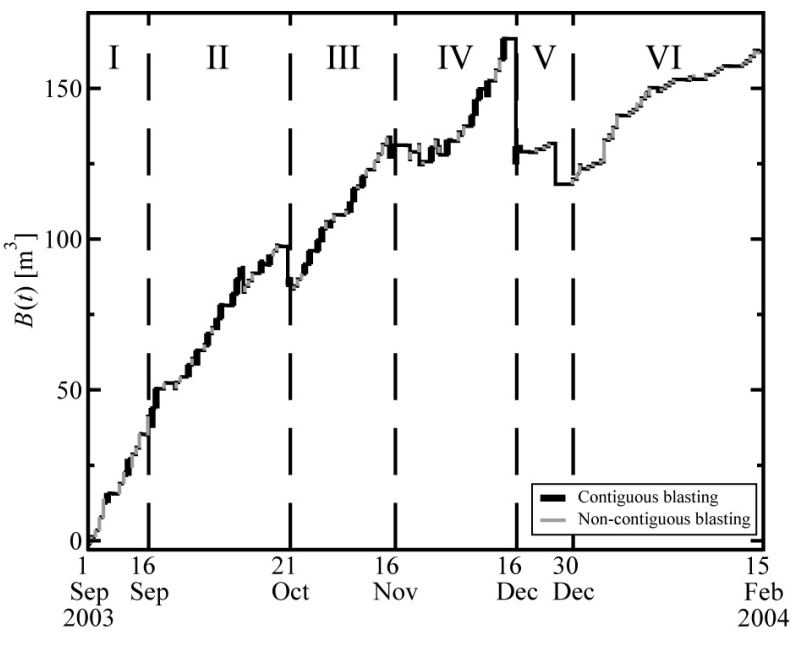

FIG. 3 Balance $B(t)$ of volume mined, backfilled and seismic potency for 166 days of mining $\Delta V=2 \cdot 10^{6} \mathrm{~m}^{3}$ volume of rock. Volume mined at each production blast is measured upwards and marked with thick lines for contiguous and thin lines for non-contiguous extraction. Backfill, measured downwards, is placed immediately after production blasts and is assumed to have $10 \%$ compaction. Seismic potency associated with each event is measured downwards

TABLE 2 The volumes mined, the rate of mining and the relevant seismic characteristics for the 6 periods of mining. Total potency released $\Sigma P=164 \mathrm{~m}^{3}$. The expected maximum magnitude, $\log P_{\text {max }}$, for the entire data set is $3.3( \pm 0.4)$ - see Figure 5. The total effective volume mined is $V_{\text {eff }}=326.7 \mathrm{~m}^{3}$, which, for $\alpha=40.76$ and $\beta=0.48$ gives $\log P_{\operatorname{maxm}}=1.8$

\begin{tabular}{lllllll}
\hline Period & I & II & III & IV & V & VI \\
\hline$V_{m},\left[\mathrm{~m}^{3}\right]$ & 497 & 799 & 599 & 641 & 47 & 684 \\
$\Delta t,[$ day $]$ & 15 & 35 & 26 & 30 & 14 & 46 \\
$V_{m} / \Delta t$ & 33 & 23 & 23 & 21 & 3 & 15 \\
$N\left(\geq P_{\min }\right)$ & 513 & 641 & 280 & 237 & 61 & 249 \\
\hline$\Sigma P,\left[\mathrm{~m}^{3}\right]$ & 12 & 37 & 18 & 70 & 17 & 10 \\
$\log P_{\max }$ & 0.4 & 1.2 & 0.9 & 1.6 & 0.9 & 0.3 \\
$\log P_{\operatorname{max0}-1}$ & 0.0 & 0.4 & 0.5 & 0.8 & 0.2 & 0.1 \\
$\alpha$ & 7.9 & 12.5 & 6.6 & 6.2 & 2.7 & 4.7 \\
$\beta$ & .52 & .49 & .47 & .45 & .39 & .49 \\
\hline $\log P_{\max }$ & 1.7 & 2.2 & 1.8 & 1.8 & 1.1 & 1.4 \\
$K_{s},[\mathrm{GPa}]$ & 51 & 15 & 44 & 26 & 124 & 88 \\
$\log P_{\operatorname{maxm}}$ & 1.6 & 1.6 & 1.9 & 1.9 & 0.7 & 2.3 \\
\hline
\end{tabular}

\subsection{Discussion}

Seismic hazard measured by the largest observed magnitude $m_{\text {maxo }}=\log P_{\text {maxo }}$, by the second largest observed magnitude $m_{\max }^{\max }-1=P_{\operatorname{maxo}}^{\max }-1$, by the $\beta$-value, by the maximum expected magnitude, $m_{\max }=\log P_{\max }$, by the total potency, by the total potency per unit of volume mined and by the total radiated energy was higher during periods of mining by blasting contiguous panels. The biggest differences between the largest expected event magnitude predicted by the power law, $\log P_{\max }$ and the largest observed one, $\log P_{\text {maxo }}$, are derived for periods of non-contiguous blasting. As time progresses, the $\beta$-value, calculated on cumulative time periods, drops and the expected maximum event size increases to $\log P_{\max }=3.3$, while the observed events remained small.

The rate of mining, $V_{m} / \Delta t$, ranged from 3 to $33 \mathrm{~m}^{3} /$ day and did not correlate with seismic hazard. Note that although the highest rate of mining was in the first period, where noncontiguous panels were blasted, seismic hazard remained low. The first larger event, $\log P>1.0$, occurred only at the end of the second period, on the 20 October 2003.

During the periods of contiguous blasting the largest events tended to occur when the net balance $B(t)$ increased by approximately $50 \mathrm{~m}^{3}$.

Seismic stiffness, $K_{s}=4 \Delta V \Sigma E /(\Sigma \mathrm{P})^{2}$ maintained its highest level during the periods of non-contiguous blasting, with the exception of Dec 16 to Dec 30, when mining was practically suspended. The maximum event size resulting from the effective volume mined in a particular time period, $\log P_{\text {maxm }}$ does not correlate with the way of blasting nor with the rate of mining, but it offered more realistic estimates. For the whole data set the $\log P_{\operatorname{maxm}}=1.8$ and for cumulative periods:

$\log P_{\text {maxm }}(1$ Sept-21Oct $)=1.6$,
$\log P_{\text {maxm }}(1$ ept-16Nov $)=1.7$,
$\log P_{\text {maxm }}(1$ ept-16Dec $)=1.8$,
$\log P_{\text {maxm }}(1$ Sept-30Dec $)=1.7$.

\section{Persistence Of Seismic Release}

Many properties of seismic activity indicate a hierarchical organization, suggesting connection among events in space and in time. A suggested mechanism is the stress transfer due to loading, including seismicity itself, and the scale invariant fracturing of rock due to self-organization (Bak et al., 2002). The short term and small spatial limit of general hierarchical phenomenon is the Omori law, which states that immediately after major shock, e.g., large seismic event or production blast, the frequency of events first jumps up and then decays with time according to power law. In fact, in deep level gold mines the most seismic energy is released within few hours after the production blasts. In some cases the rate of convergence and associated seismic activity that follows the daily production blasts increases during the working week. This suggests that the stress relaxation processes may be slower than the cycle of face advance (Hodgson, 1967; McGarr, 1971). Kijko et al. (1993) detected similarities in probability patterns between clusters of seismic activity separated by more than 1000 meters, suggesting that the range of spatial correlations may be comparable with the size of the mine. These and other factors may generate long term correlations in the system that result in non-periodic cyclical patterns of seismic release. This long-term memory leads to persistence of the process which means that quiet days tend to be followed by quiet days and active days by active days. Such correlations may be destroyed by very large event, that tends to de-correlate part of, or the whole system almost instantaneously, or by change in the loading conditions, in which case the change in the response of the system would be gradual.

Detection of long-term correlations in data is not simple task. Long term correlations can be masked by trends: uncorrelated data may, in the presence of long term trends, look like correlated data and, conversely, long-term correlated data may look like uncorrelated data influenced by trend. It is therefore advisable to detrend the data set before analysis. It is also advisable to perform the analysis on the cumulative functions, because it suppresses the high frequency oscillations that may obscure the long-term behaviour.

A frequently used method for detection of long term correlations in data is the rescaled range or R/S analysis (Mandelbrot and Wallis, 1969), which is based on hydrological work by Hurst (1951). Applied to the potency, the R/S 

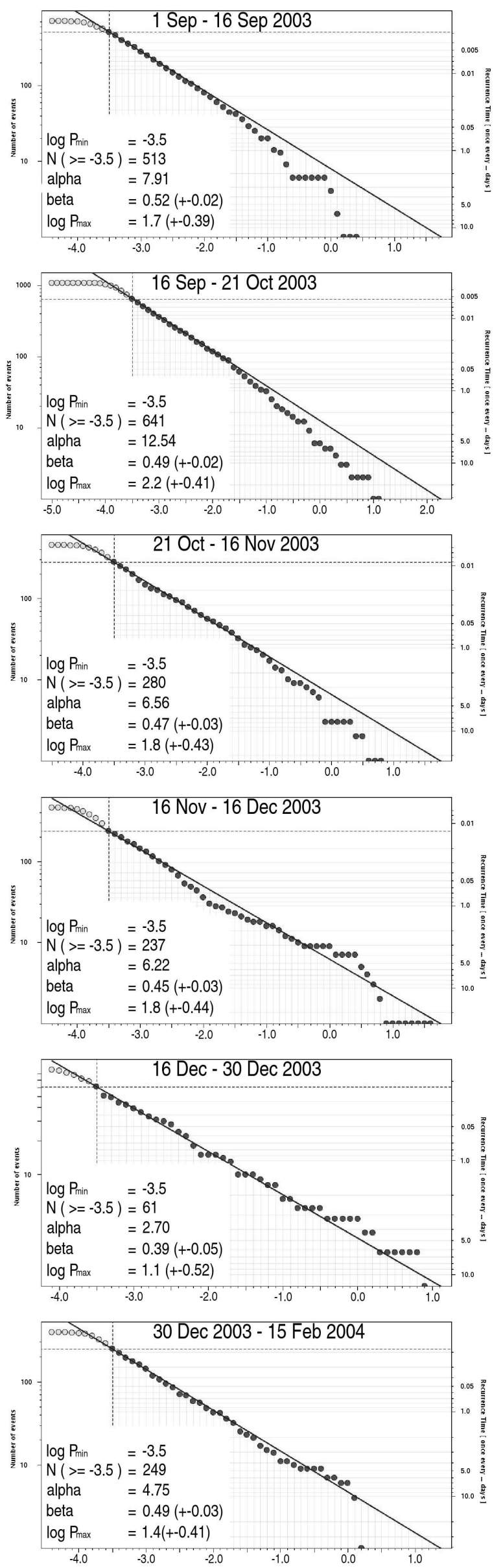

FIG. 4 Log potency-frequency distributions for each of the time periods indicated in Figure 3
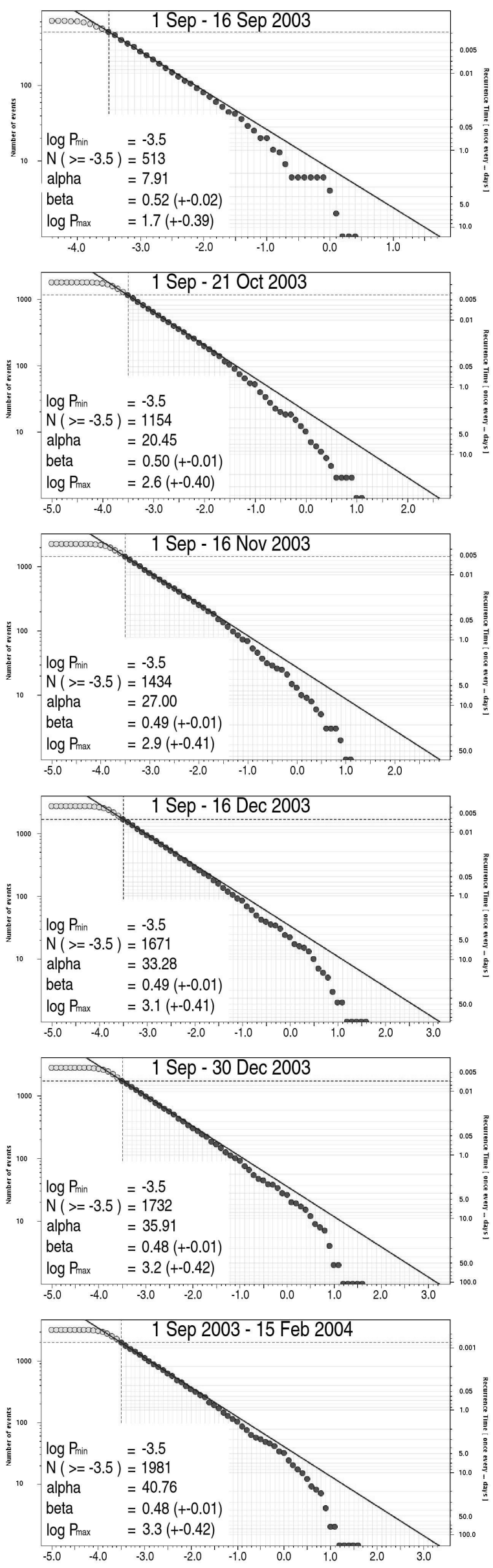

FIG. 5 Log potency-frequency distributions for the cumulative periods starting on 1 Sept' 03 
method calculates the standard deviation $s_{d}(\Delta t)$ of the potency produced over the interval $\Delta t$ and the range $R$ of deviation $d$, $R(\Delta t)=\max (d)-\min (d)$, of the cumulative potency function $P(\Delta t)=\Sigma_{t k<t} P\left(t_{k}\right) \theta\left(t-t_{k}\right)$ from its average trend approximated by the straight line fitted to this function. For different time intervals there are different straight line fits and different ranges of deviations. The ratio $R / s_{d}$ averaged over all intervals of length $\Delta t$ is called the rescaled range and for large $\Delta t$ it scales as

$$
\frac{R(\Delta t)}{s_{d}(\Delta t)}=(a \cdot \Delta t)^{H}
$$

where $H$ is the similarity index or the Hurst exponent and $a$ is scale factor. Having determined $H$ and $a$ for the entire catalog of available data and assuming that the standard deviation $s_{d}(\Delta t)$ will remain constant, one can estimate the expected range of the potency generated by the system $R(\Delta t)$ as function of the span $\Delta T$ into the future (Lomnitz, 1994)

$$
R(\Delta T)=s_{d}(\Delta t)(a \cdot \Delta T)^{H}
$$

The R/S method of estimating the Hurst exponent is susceptible to spurious detection of the apparent long term correlation that is an artifact of non-stationarity. An improved variant of this method called Detrended Fluctuation Analysis (DFA) developed by Peng et al. (1994) and described in Appendix A.1 avoids this problem (Huetal.,2001). A procedure to forecast the range of potency produced $R(\Delta T)$ to be expected within a period of $\Delta T$ days after the last measurement, based on DFA, is given in Appendix A.2. The Hurst exponent offers an interesting interpretation (Mandelbrot,2002; Goltz,1997):

- $H=0.5$ - lack of correlations, characteristic of white noise, it means that the successive steps in the underlying time series are independent - a random walk. The best prediction is the last measured value.

- $H>0.5$ - positive corelations, it means the local trend will persist. The best prediction is based on the extrapolation of the trend. For most natural processes, like earthquakes or rainfall, $H=0.72 \pm 0.2$.

- $H<0.5$ - anti-correlations, it means the reversal of the trend or anti-persistence. The best prediction tends to the mean value.

It is frequently assumed that the Hurst exponent and the fractal dimension $D$, that measure the roughness of the process, are always linearly related. In principle, however, fractal dimension and Hurst exponent are independent of each other: fractal dimension is a local property, and longmemory dependence is a global characteristic. Nevertheless, for self-similar processes in $n$-dimensional space, where the statistical properties of subsets are the same as for the entire data set, the local properties are reflected in the global ones and $D+H=n+1$ (Mandelbrot, 2002). For a one-dimensional space then, $D=2-H$, but only for self similar data sets.

A larger Hurst exponent means a lower fractal dimension which is characteristic of a smoother process. Likewise, a smaller Hurst exponent means a higher fractal dimension and correspondingly a rougher process. For small earthquakes, whose spatial structure in a given volume is fractal, the fractal dimension is expected to be correlated with the $b$-value of the frequency-magnitude distribution as $\mathrm{D} \simeq 2 b$ (Aki, 1981; Wyss et al., 2004). For the frequency-potency distribution, where $b=1.5 \beta$ the fractal dimension would scale as $\mathrm{D}=3 \beta$ and, consequently, for one-dimensional space, $H=2-3 \beta$.

Figure 6 shows Hurst graphs of the cumulative potency and the balance $B(t)$ for the entire data set of 166 from 1 Sept 03 to $15 \mathrm{Feb}^{\prime} 04$. The linear character of the Hurst graph indicates a power law scaling and its slope is the Hurst exponent. The exponent for cumulative potency is high, $H=0.825$ and, if
Data set: 1 Sep 2003 - 15Feb 2004

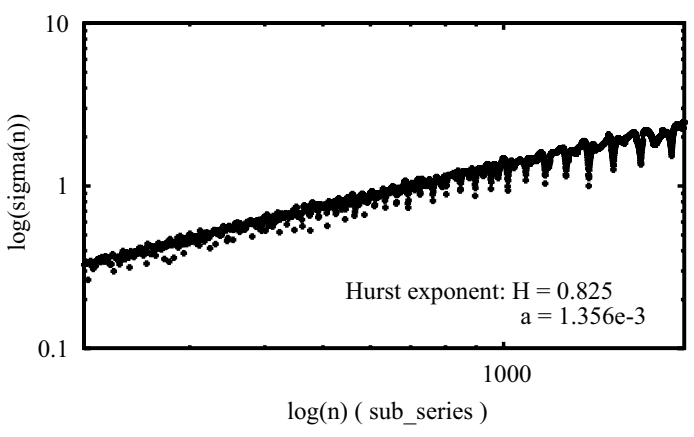

Data set: 1 Sep 2003 - 15Feb 2004

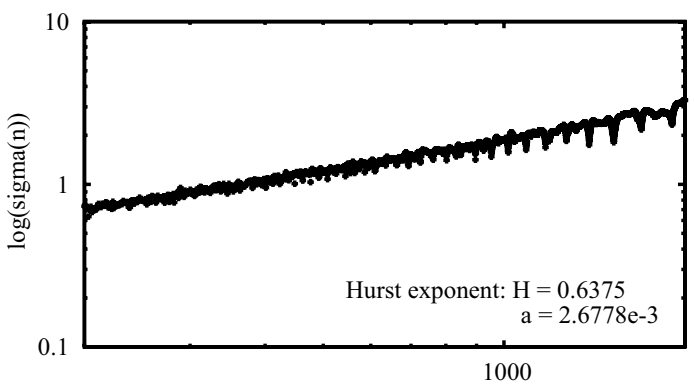

$\log (\mathrm{n})$ ( sub_series )

FIG. 6 The Hurst graphs of the cumulative potency (top) and of the balance function $B(t)$ (bottom), for the whole period, 1 Sept'03 to $15 F^{\prime}$ 'b'04

correct, would indicate a well established persistent behaviour, implying that the current trend of potency production will tend to continue regardless of short-term changes to the way production blasting. The Hurst exponent for the balance is lower, $H=0.637$, indicating a rougher process, but sufficiently above 0.5 to expect a degree of persistence. The roughness of the balance, in relation to potency, may result from the fact that it incorporates data on two competing processes, namely, on excavation and on resulting seismic deformation.

Figure 7 shows the cumulative potency and the balance $B(t)$ for the whole data set, the trend and the forecasted range of these parameters for 7, 30, 60 and 90 days ahead. For 30 days ahead, the estimated range of potency produced is $17.2 \mathrm{~m}^{3}$ which, if delivered in a single seismic event, corresponds to $\log P=1.2$. For 60 days ahead, the forecasted range of potency is $30.5 \mathrm{~m}^{3}(\log P=1.5)$ and for 90 days $42.6 \mathrm{~m}^{3}$ $(\log P=1.6)$. Note that the mean recurrence time for events with $\log P \sim 1.2, \log P \sim 1.5$ and $\log P \sim 1.6$, given by the potency-frequency statistics for the whole period, are 15, 21 and 23 days respectively - see Figure 5 . The forecasted range for the balance is also lower than the observed one and over the next 30 days it is forecasted at $19.05 \mathrm{~m}^{3}$, over 60 days at $29.64 \mathrm{~m}^{3}$ and over 90 days at $38.38 \mathrm{~m}^{3}$.

For a few months after $15 \mathrm{Feb}^{\prime} 04$, mining continued by blasting predominantly a single panel or two non-contiguous panels. Experience with sequential grid mining shows that the larger events are generated rather later in the cycle, when mining spans gets bigger. In this case, however, the subdued seismic response persisted. The following four largest events recorded in the area within three months after $15 \mathrm{Feb}^{\prime} 04$ were clearly smaller than predicted by the frequency-potency distribution and even smaller than forecasted by the Hurst statistics: $\log P=1.2$ on March $14, \log P=1.1$ on March 26, $\log P=1.2$ on May $03, \log P=1.0$ on June 05 - see Figure 2 . 


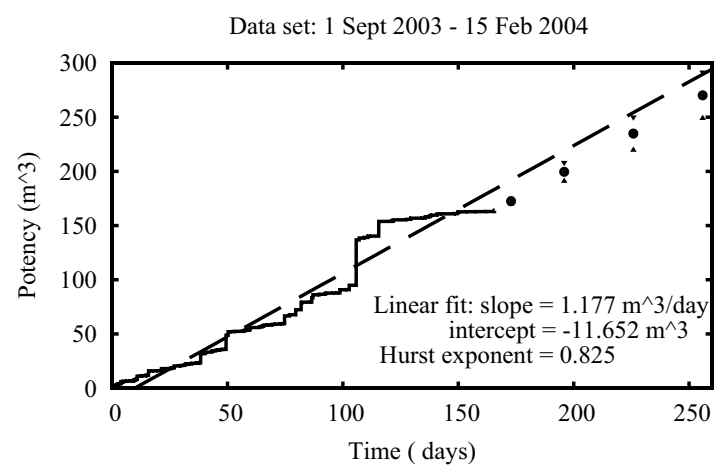

Data set: 1 Sept 2003 - 15 Feb 2004

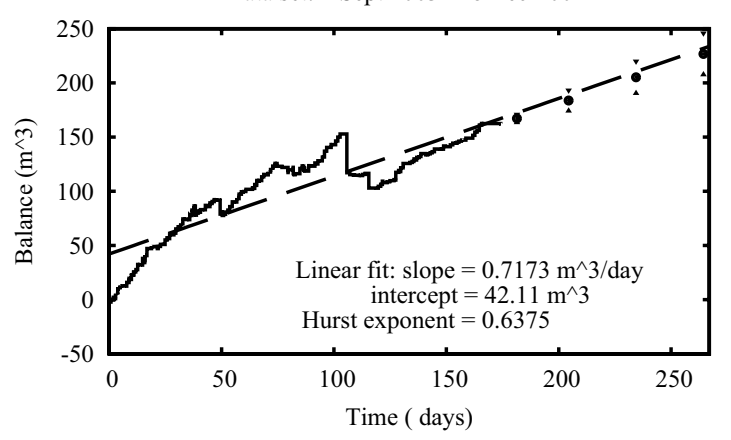

FIG. 7 The cumulative potency (top) and the balance $B(t)$ (bottom) for the whole period of 166 days. The straight line represent trend imposed by the existing data and the little triangles indicate the expected range of values at 7 , 30,60 and 90 days ahead

\section{SUMMARY}

It has been shown that the short term hazard associated with spatially disordered non-contiguous blasting of mining panels was lower than when blasting proceeded contiguously, irrespective of the rate of mining. Non-contiguous blasting at a lower mining rate generated even more favorable conditions. These results confirmed the previous observations of seismic rockmass response to production blasting associated with long-wall mining. The data sets also show the presence of long-term correlations, indicating the tendency for the local trend of lower seismic hazard to persist. It may be that mining scenarios that induce spatial heterogeneity prevent smoothing, de-correlate the system and are less likely to develop larger dynamic instabilities.

\section{ACKNOWLEDGMENT}

This research was part of the project "Optimization of the Routine Seismic Hazard Rating System" sponsored by Anglogold Ashanti.

\section{REFERENCES}

Abercrombie, R.E. (1996) The magnitude-frequency distribution of earthquakes recorded with deep seismometers at cajon pass., Tectonophysics, 261, 1-7.

Aki, K. (1967) Scaling law of seismic spectrum, Journal of Geophysical Research, 72, 1217-1232.

Aki, K. (1969) Analysisof the seismic coda of the local earthquakes as scattered waves, Journal of Geophysical Research, 74, 615-631.

Aki, K. (1981) A probabilistic synthesis of precursory phenomena, in Earthquake Prediction: An International Review, edited by D.W. Simpson and P.G. Richards, Maurice Eving, pp. 566-574, American Geophysical Union, Washington D. C.

Aki, K. (1987) Magnitude-frequency relation for small eartqquakes: A clue to the origin of fmax of large earthquakes, Journal of Geophysical Research, 92(B2), 1349-1355.

Amitrano, D. (2003) Brittle-ductile transition and associated seismicity: Experimental and numerical studies and relationship with the b-value, Journal of Geophysical Research, 108 (B1)(2044), 1-15, doi:10.1029/2001JB000680.
Bak, P., Christensen, K., Danon, L. and Scanlon, T. (2002) Unifying scaling law for earthquakes, Phys. Rev. Lett., 88(178501).

Ben-Menahem, A. and Singh, S.J. (1981) Seismic Waves and Sources, SpringerVerlag, New York

Ben-Zion, Y. (2001) On quantification of the earthquake source, Seismological Research Letters, 72(2), 151-152.

Ben-Zion, Y. and Zhu, L. (2002) Potency-magnitude scaling relation for southern california earthquakes with $1.0<\mathrm{m}<7.0$, Geophysical Journal International, 148, F1-F5.

Goltz, C. (1997) Fractal and Chaotic Properties of Earthquakes, no. 77 in Lecture Notes in Earth Science, Springer-Verlag, Berlin Heidelberg.

Gutenberg, B. (1945a) Amplitudes of surface waves and magnitudes of shallow earthquakes, Bulletin of the Seismological Society of America, 35, 3-12.

Gutenberg, B. (1945b) Amplitudes of p, pp and s and magnitudes of shallow earthquakes, Bulletin of the Seismological Society of America, 35, 57-69.

Gutenberg, B. and Richter, C.F. (1956) Earthquake magnitude, intensity, energy and acceleration., Bulletin of the Seismological Society of America, 46, 105-145.

Hanks, T.C. and Kanamori, H. (1979) A moment magnitude scale, Journal of Geophysical Research, 84, 2348-2350.

Haskell, N. (1964) Total energy and energy spectral density of elastic wave radiation from propagating faults, Bulletin of the Seismological Society of America, 56, 1811-1842.

Heaton, T.H. and Heaton, R.E. (1989) Static deformations from point sources and force couples located in welded elastic poissonian half-spaces: Implications for seismic moment tensors., Bulletin of the Seismological Society of America, 79(3), 813-841.

Hodgson, K. (1967) The behaviour of the failed zone ahead of a face, as indicated by continuous seismic and convergence measurements, Tech. Rep. 31/61, Chamber of Mines Research Organization, Republic of South Africa.

Hu, K., Ivanov, P.C., Chen, Z., Carpena, P. and Stanley, H.E. (2001) Effect of trends on detrended fluctuation analysis, Physical Review E, 64(011114).

Hurst, H.E. (1951) Long-term storage capacity of reservoirs, Transactions of the American Society of Civil Engineers, 116, 770-808.

Izutani, Y. (1984) Source parameters relevant to heterogeneity of a fault plane, J. Phys. Earth, 32, 511-529.

Kanamori, H. (1977) The energy release in great earthquakes, Journal of Geophysical Research, 82, 2981-2987.

Kanamori, H. and Anderson, D.L. (1975) Theoretical basis of some empirical relations in seismology, Bulletin of the Seismological Society of America, 65(5), 1073-1095.

Keilis-Borok, V.I., Bessanova, E.N., Gotsadze, O.D., Kirilova, I.V., Kogan, S.D., Kikhtikova, T.I., Malinovskaya, C.N., Pavola, G.I. and Sarskii, A.A. (1960) Investigation of the mechanism of earthquakes (english translation), American Geophysical Union, Consultants Bureau, New York.

Kijko, A., Funk, C.F. and Brink, A.vZ. (1993) Identification of anomalous patterns in time dependent mine seismicity, in Proceedings of the 3rd International Symposium on Rockburst and Seismicity in Mines, Kingston, Ontario, Canada, edited by R. P. Young, pp. 205-210.

King, G.C.P. (1978) Geological faults, fractures, creep and strain, Phil. Trans. Royal Society London, 288, 197-212.

Lomnitz, C. (1994) Fundamentals of Earthquake Prediction, John Wiley and Sons.

Mandelbrot, B.B. (2002) Gaussian Self-Affinity and Feactals: Globality, The Earth, 1 /f noise, and R/S, Springer.

Mandelbrot, B.B. and Wallis, J.R. (1969) Robustness of the rescaled r/s in the measurement of noncyclic long run statistical dependence, Water Resources Research, AGU, 5, 967-988.

McGarr, A. (1971) Stable deformation of rock near deep-level tabular excavations, Journal of Geophysical Research, 76(29), 7088-7106.

McGarr, A. (1976a) Seismic moments and volume changes, Journal of Geophysical Research, 81, 1487-1494.

Mendecki, A.J. (1993) Real time quantitative seismology in mines: Keynote address, in Proceedings of the 3rd International Symposium on Rockbursts and Seismicity in Mines, Kingston, Ontario, Canada, edited by R.P. Young, pp. 287-295, Balkema, Rotterdam.

Mendecki, A.J. (2001) Data-driven understanding of seismic rockmass response to mining: Keynote address, in Proceedings of the 5th International Symposium on Rockbursts and Seismicity in Mines, Johannesburg, South Africa, edited by G. van Aswegen, R. J. Durrheim, and W. D. Ortlepp, pp. 1-9, South African Institute of Mining and Metallurgy.

Mendecki, A.J., van Aswegen, G. and Mountfort, P. (1999) A guide to routine seismic monitoring in mines, in A Handbook on Rock Engineering Practice for Tabular Hard Rock Mines, edited by A. J. Jager and J. A. Ryder, chap. 9, pp. 287-309, The Safety in Mines Research Advisory Committee, Johannesburg. 
Mogi, K. (1962) Magnitude frequency relations for elastic shocks accompanying fractures of various materials and some related problems in earthquakes, Bulletin Earthquake Research Institute Tokyo University, 40(831-853).

Mori, J. and Abercombie, R.E. (1997) Depth dependence of earthquake frequency-magnitude distribution in california: Implications for rupture initiation, Journal of Geophysical Research, 102(B7), 15,081-15,090.

Peng, C.K., Buldyrev, S.V., Havlin, S., Simons, M., Stanley, H.E. and Goldberger, A.L. (1994) Mosaik organization of dna nucleotides., Physical Review E, E49, 1685-1689.

Real, C.R. and Teng, T.L. (1973) Local richter magnitude and total signal duration in southern california, Bulletin of the Seismological Society of America, 63, 1809-1828.

Richter, C.F. (1935) An instrumental earthquake magnitude scale, Bulletin of the Seismological Society of America, 25, 1-32.

Scholz, C.H. (1968) The frequency-magnitude relation of microfracturing in rock and its relation to earthquakes, Bulletin of the Seismological Society of America, 58(1), 399-415.

Spottiswoode, S.M. and McGarr, A. (1975) Source parameters of tremors in a deep-level gold mine., Bulletin of the Seismological Society of America, 65(1), 93-112.

Thatcher, W. and Hanks, T.C. (1973) Source parameters of southern california earthquakes, Journal of Geophysical Research, 78, 8547-8574

van Aswegen, G. and Butler, A.G. (1993) Applications of quantitative seismology in south african gold mines, in Proceedings of the 3rd International Symposium on Rockbursts and Seismicity in Mines, Kingston, Ontario, Canada, edited by R.P. Young, pp. 261-266, Balkema, Rotterdam, iSBN 9054103205.

van Aswegen, G. and Mendecki, A.J. (1999) Mine layout, geological features and seismic hazard., Final report, SIMRAC, Department of Minerals and Energy.

Wiemer, S. and Wyss, M. (1997) Mapping the frequency-magnitude distribution in asperities: An improved technique to calculate recurrence times, Journal of Geophysical Research, 102(B7), $15,115-15,128$.

Wyss, M. and Brune, J.N. (1968) Seismic moment, stress and source dimensions for earthquakes in the california-nevada region, Journal of Geophysical Research, 73, 4681-4694.

Wyss, M., Sammis, C.G., Nadeau, R.M. and Wiemer, S. (2004) Fractal dimension and b-value on creeping and locked patches of the san andreas fault near parkfield, california, Bulletin of the Seismological Society of America, 94(2), 410-421.

\section{APPENDIX}

\section{Algorithm 1:}

Detrended Fluctuation Analysis and the Hurst Exponent

1. Divide a given time series $X_{j}$ of length $L$ into $n_{s}$ subseries of length $n: X_{j, m}=X_{(m-1) n+j} ; m=1,2, \ldots, n_{s}$.

2. Create the cumulative time series $Y_{i, m}=\Sigma_{j=1}^{i} X_{j, m}$ for $i=1, \ldots, n$ and $m=1,2, \ldots, n$.

3. For each sub-series $\bar{Y}_{i, m}$ :

- fit the least squares line, $Y(x)=a_{m} x+b_{m}$ to the points: $\left(1, Y_{1, m}\right),\left(2, Y_{2, m}\right), \ldots,\left(n, \mathrm{Y}_{n, m}\right)$

- calculate the standard deviation of the detrended time series

$$
s_{d}(m)=\sqrt{\frac{1}{n} \sum_{i=1}^{n}\left(Y_{i, m}-a_{m} i-b_{m}\right)^{2}} .
$$

4. Average the standard deviations over all sub-series for the current value of $n$

$$
\overline{\mathrm{s}_{d}}(n)=\frac{1}{n_{s}} \sum_{m=1}^{n_{s}} s_{d}(m) .
$$

5. Fit a straight line to $\log \bar{s}_{d}(n)$ vs $\log (n): \log \left[\bar{s}_{d}(n)\right]=$ $H \cdot[\log (n)+\log (a)]$. A linear dependence of $\log \left(\bar{s}_{d}\right)$ on $\log (n)$ indicates a power law scaling $\bar{s}_{d}(n)=(a \cdot n)^{H}$, where $H$ is the Hurst exponent and $a$ is a constant scale factor

\section{Algorithm 2:}

\section{Estimating the Range of the Future Potency}

1. Compute the Hurst exponent $H$ and the scaling parameter $a$.

2. Fit a straight line $c \cdot i+d$ to the whole cumulative potency series $Y_{i}$

3. Convert the time interval $\Delta T$ of the forecast into number of terms $\mathrm{n}$ in the cumulative potency time series (NB: the terms of the input cumulative potency time series are supposed to be equally spaced in time).

4. Use the scaling relation $s_{d}(n)=(a \cdot n)^{H}$ to estimate the average standard deviation of the detrended series for this value of $n$.

5. Extrapolate the cumulative potency series beyond the last term $Y_{N}$ by means of the slope of $Y_{N}+m=$ $Y_{N}+c \cdot \Delta T$ (using the slope $c$ of the global linear fit to the cumulative series).

6. Look up from the table of confidence level intervals in terms of standard deviations. The factor $f$ corresponds to the required accuracy of the forecast, e.g., factor $f=1$ gives $68.3 \%$ confidence level, factor $f=2$ gives $95.4 \%$ confidence level. Compute the spread, spread $=f \cdot \bar{s}_{d}$.

7. The expected minimum and maximum values of the cumulative potency produced for the said period of time are obtained as $Y_{N}+n \pm$ spread respectively. 